\title{
Invaded cluster dynamics for frustrated models
}

\author{
Giancarlo Franzese, ${ }^{1, *}$ Vittorio Cataudella, ${ }^{1, *}$ and Antonio Coniglio ${ }^{1,2, *}$ \\ ${ }^{1}$ INFM, Unità di Napoli, Napoli, Italy \\ ${ }^{2}$ INFN, Sezione di Napoli, Napoli, Italy
}

(Received 2 July 1997)

\begin{abstract}
The invaded cluster (IC) dynamics introduced by Machta et al. [Phys. Rev. Lett. 75, 2792 (1995)] is extended to the fully frustrated Ising model on a square lattice. The properties of the dynamics that exhibits numerical evidence of self-organized criticality are studied. The fluctuations in the IC dynamics are shown to be intrinsic of the algorithm and the fluctuation-dissipation theorem is no longer valid. The relaxation time is found to be very short and does not present a critical size dependence. [S1063-651X(97)07012-8]
\end{abstract}

PACS number(s): 64.60.Lx, 75.40.Mg, 64.60.Ak, 64.60.Fr

\section{INTRODUCTION}

Recently, a nonstandard cluster Monte Carlo (MC) dynamics, the invaded cluster (IC) dynamics, based on invaded percolation has been introduced by Machta et al. for the ferromagnetic Ising model [1]. The IC dynamics is based on the Kastelein-Fortuin-Coniglio-Klein (KF-CK) cluster formulation of the Ising model [2] and has been shown to be even more efficient than the Swendsen-Wang (SW) dynamics [3] in equilibrating the system at the critical temperature. The IC dynamics has the advantage that the value of the critical temperature does not need to be known a priori. In fact, the dynamics itself drives the system to the critical region as in self-organized critical (SOC) systems.

The aim of this paper is to extend the IC dynamics to frustrated systems where KF-CK clusters percolate at a temperature $T_{p}$ higher than the critical temperature $T_{c}$. In particular, we will consider the fully frustrated (FF) Ising model on a square lattice where it has been shown numerically [4] that $k_{B} T_{p} / J \simeq 1.69\left(k_{B}\right.$ is the Boltzmann constant and $J$ is the strength of the interaction) and $T_{c}=0$. We will use two definitions of clusters. The first definition is based on the KF-CK clusters. In this case the IC dynamics leads to a SOC percolating state at temperature $T_{p}$ corresponding to the percolation of KF-CK clusters. The second definition is more general [5] and reduces to the cluster of Kandel, Ben-Av, and Domany [6] in the FF Ising model. In this case the IC dynamics leads to a SOC state at the thermodynamical critical temperature $T_{c}=0$.

In Sec. II we review the definition and the results of IC dynamics on the ferromagnetic Ising model. Then we extend the IC dynamics to the FF Ising model using the KF-CK cluster in Sec. III and the Kandel-Ben-Av-Domany (KBD) clusters in Sec. IV, where we study also the equilibrium relaxation of the proposed dynamics. We present conclusions in Sec. V.

\section{IC DYNAMICS FOR THE FERROMAGNETIC ISING MODEL}

The rules that define the IC dynamics for the ferromagnetic Ising model are very simple. Let us start from a given

\footnotetext{
*Permanent address: Dipartimento di Scienze Fisiche, Università, “'Federico II,', Mostra d'Oltremare Pad. 19, 1-80125 Napoli, Italy.
}

spin configuration. As the first step all the pairs of nearestneighbor $(\mathrm{NN})$ spins are ordered randomly. Then, following the random order, a bond is activated between the NN spin pairs only if the two spins satisfy the interaction. The set of activated bonds partitions the lattice into clusters that are classified every time a new bond is activated. When one of the clusters spans the system the procedure is stopped and the spins belonging to each cluster are reversed all together with probability $1 / 2$. The spin configuration obtained can be used as a starting point for the next application of the dynamical rule. Successively, the algorithm has been generalized to different stopping rules $[7,8]$ and to different models (the Potts model [7] and the Widom-Rowlinson fluid [9]).

The rationale behind this rule is the well-known mapping between the ferromagnetic Ising model and the correlated bond percolation. [2] In this framework bonds are introduced in the ferromagnetic Ising model between parallel NN pairs of spins with probability $p=1-\exp (-2 \beta J)$, where $\beta=1 / k_{B} T$. The clusters (KF-CK clusters), defined as maximal sets of connected bonds, represent sets of correlated spins and percolate exactly at the critical temperature $T_{p}=T_{c}$. The well-known $\mathrm{SW}$ cluster dynamics [3] uses these clusters to sample very efficiently the phase space at any temperature. In this dynamics at each MC step the KF-CK clusters are constructed and the spins belonging to each cluster are reversed altogether with probability $1 / 2$. This produces a new spin configuration on which a new cluster configuration can be built. The IC dynamics is very similar to SW dynamics and differs only in the way clusters are constructed. Since the dynamics rule introduced by Machta et al. builds clusters that, by definition, percolate through the system, it is expected that the average properties of the clusters are the same as the KF-CK clusters at the critical temperature. In fact, in Ref. [1] it has been shown that the ratio of activated bonds to satisfied interactions, in the large- $L$ limit ( $L$ is the linear lattice size), is very close to the critical probability $p_{c}=1-\exp \left(-2 J / k_{B} T_{c}\right)$ with which KF-CK clusters are constructed and it has a vanishing standard deviation. Furthermore, the estimated mean energy also tends in the large- $L$ limit to a value very close to the critical equilibrium value $E\left(T_{c}\right)$ with the finite-size behavior expected in the ferromagnetic Ising model at the critical point. On the other hand, the energy fluctuation $C=\left\langle E^{2}\right\rangle-\langle E\rangle^{2}$ is not related to the specific heat. In fact, it has been found that $C$ diverges 
linearly with $L$ and not logarithmically as the specific heat does. The latter result points to the fact that the IC dynamics does not sample the canonical ensemble in finite volume and therefore energy fluctuations and specific heat are no longer related by the fluctuation-dissipation theorem. Since the fluctuation $C^{1 / 2} / L^{2} \rightarrow 0$ for $L \rightarrow \infty$, it is generally assumed that in the thermodynamic limit the IC ensemble is equivalent to the canonical ensemble even if a rigorous proof is still lacking.

\section{IC DYNAMICS FOR THE FF ISING MODEL WITH KF-CK CLUSTERS}

We now extend the IC dynamics to frustrated systems where the KF-CK clusters percolate at a temperature $T_{p}$ that is higher than the critical one $T_{c}$. In particular, we will consider the FF Ising model on a square lattice where [10] $T_{p} \simeq 1.69$ and $T_{c}=0$ [4]. The FF Ising model is defined by the Hamiltonian

$$
H=-J \sum_{\langle i j\rangle}\left(\epsilon_{i, j} S_{i} S_{j}-1\right),
$$

where $S_{i}$ takes the values \pm 1 and $\epsilon_{i, j}$ assumes the value -1 on even columns and +1 otherwise.

In the simplest extension of the IC dynamics we introduce bonds at random between spins satisfying the interaction (i.e., $\epsilon_{i j} S_{i} S_{j}=1$ ) until a spanning cluster is found. As in the ferromagnetic case, at this point the spins belonging to each cluster are reversed altogether with probability $1 / 2$. The procedure is iterated until equilibrium is reached. We have done simulations performing measurements over $5 \times 10^{3} \mathrm{MC}$ sweeps after discarding the first $10^{3}$ for equilibration for systems with sizes $L$ ranging from 16 to 300 and over $3.75 \times 10^{3}$ MC sweeps after discarding the first 750 for $L=350$.

In Fig. 1(a) we show as functions of the system size $L$ the ratio of activated bonds $N_{b}$ to the satisfied interaction $N_{s}=2 L^{2}-E / 2$ (where $E$ is the energy), $\langle f\rangle=N_{b} / N_{s}$, and in Fig. 1(b) the density of energy $\epsilon=E / L^{2}$. Assuming the size dependences $\langle f\rangle=\langle f\rangle_{\infty}-A / L$ and $\epsilon=\epsilon_{\infty}+A / \ln (L)$ we find $\langle f\rangle_{\infty}=0.698 \pm 0.008$ and $\epsilon_{\infty}=1.24 \pm 0.01$, which are very close to the values $p_{c}=1-e^{-2 / T_{p}} \simeq 0.694$ and $\epsilon\left(T_{p}\right)=1.234$ at the temperature $T_{p} \simeq 1.69$. The errors on $\langle f\rangle$ and $\epsilon$ decrease with increasing $L$, meaning that the $\langle f\rangle$ and the $\epsilon$ distributions become sharp when $L \rightarrow \infty$.

The results obtained show that the dynamics has not driven the system into the critical thermodynamical state at $T=0$, but to the percolation critical state at $T=T_{p}$. In order to study the convergence as a function of $L$ we have extracted for the IC dynamics at each $L$ an effective temperature $T_{I C}(L)$ (see Table I) by using $\langle f\rangle=1-\exp \left(-2 / T_{I C}\right)$; then we have compared the IC energy density to the analytical energy density $\epsilon\left(T_{I C}\right)$ at $T_{I C}$ [Fig. 1(b)]. This analysis clearly show that the $L \rightarrow \infty$ limit is reached in the IC dynamics very slowly. In particular, the energy is systematically larger than that obtained by SW dynamics. Furthermore, since the dynamics builds, by definition, percolating clusters, the mean cluster size $S / L^{2}$ of IC clusters diverges as $S / L^{2}=A L^{\gamma_{p} / \nu_{p}}$, where $\gamma_{p} / \nu_{p}=1.78 \pm 0.11$ is a good approximation of the exponent $\gamma_{p} / \nu_{p}=1.792$ of random bond

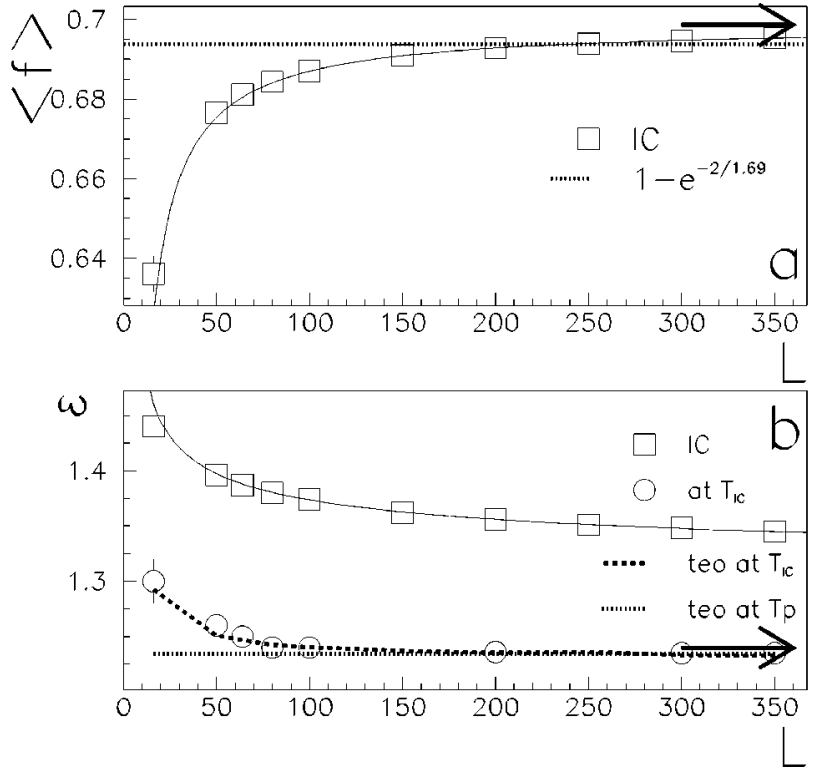

FIG. 1. KF-CK clusters: (a) ratio of activated bonds to the satisfied interaction $\langle f\rangle$ and (b) the energy density $\epsilon$ versus system size $L$ for a square FF system. Squares are the IC dynamics output, the superimposed lines are the plot of the fit functions $\langle f\rangle=\langle f\rangle_{\infty}-A / L$ (with $\langle f\rangle_{\infty}=0.698 \pm 0.008$ and $A=1.2 \pm 0.2$ ), and $\epsilon=\epsilon_{\infty}+A / \ln (L) \quad$ (with $\epsilon_{\infty}=1.24 \pm 0.01$ and $A=0.62 \pm 0.07$ ) for $L \geqslant 100$; circles are the energy density values of a standard MC dynamics at the estimated temperature $T_{I C}$ in Table I; the dashed line is the analytical energy density for the model at $T_{I C}$; the dotted line is the asymptotic value at $T=1.69 \simeq T_{p}$; the arrows show the asymptotic values estimated by the fits. Where not shown, the errors are included in the symbols; all quantities are dimensionless [10].

percolation [11] and $A=0.022 \pm 0.013$ (Fig. 2). This result is in excellent agreement with the behavior found for KF-CK clusters for both the critical exponent and the prefactor $A$ [4]. This critical behavior drives also energy and magnetization fluctuations in a critical regime, i.e., $\left\langle E^{2}\right\rangle-\langle E\rangle^{2} \sim L^{2.9}$ and $\left\langle M^{2}\right\rangle-\langle M\rangle^{2} \sim L^{3.8}$ (Fig. 3). This result is in strong contrast with the behavior of specific heat and magnetic susceptibility at $T=T_{p}$, which behave as $L^{2}$. In fact, $T_{p}$ is not a critical thermodynamic temperature and both the specific heat and magnetic susceptibility are finite at $T=T_{p}$. This again stresses the fact that the IC dynamics does not sample the canonical ensemble in a finite volume and shows that the

TABLE I. KF-CK clusters: numerical estimates of $T_{I C}(L)$ [10].

\begin{tabular}{ccc}
\hline \hline$L$ & $T_{I C}(L)$ & Error \\
\hline 16 & 1.98 & 0.04 \\
50 & 1.77 & 0.02 \\
64 & 1.75 & 0.01 \\
80 & 1.73 & 0.01 \\
100 & 1.72 & 0.01 \\
150 & 1.703 & 0.007 \\
200 & 1.695 & 0.007 \\
250 & 1.689 & 0.005 \\
300 & 1.686 & 0.004 \\
350 & 1.682 & 0.004 \\
\hline \hline
\end{tabular}




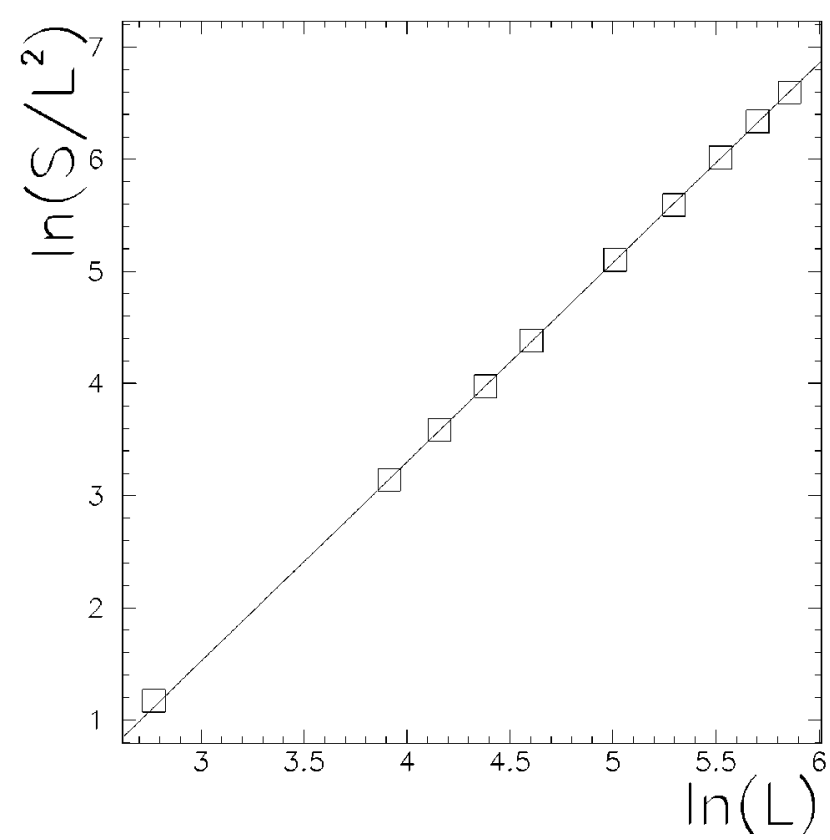

FIG. 2. KF-CK clusters: mean cluster size per spin $S / L^{2}$ for IC dynamics for a square FF system with $L=16-350$. We show the fit over data points for $L \geqslant 150$ with the best-fit parameters given in the text $\left(A=0.022 \pm 0.013\right.$ and $\left.\gamma_{p} / \nu_{p}=1.78 \pm 0.11\right)$. The errors are included in the symbols; all quantities are dimensionless [10].

spin configurations selected by the IC dynamics with a finite probability correspond to a much larger range of energies and magnetization than in any ordinary dynamics.

It is interesting to note that the straightforward application of the IC dynamics to more complex systems such as the
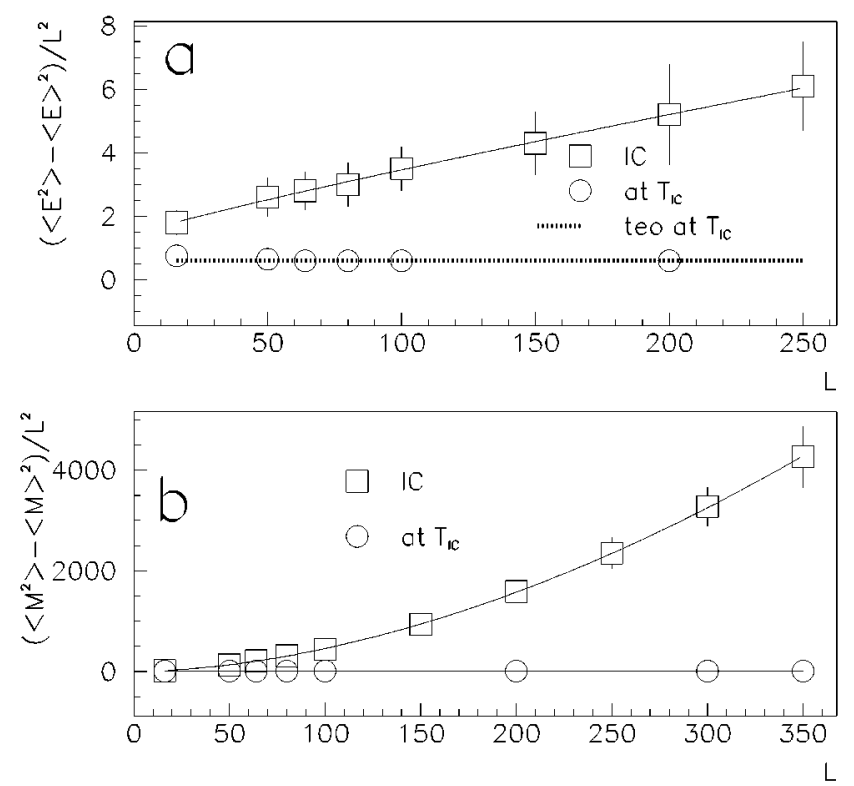

FIG. 3. KF-CK clusters: density of (a) the energy fluctuation and (b) the magnetization fluctuation for a square FF system. Squares are the fluctuations for IC dynamics; circles are the fluctuation values of a standard MC dynamics at the estimated temperature $T_{I C}$ in Table I; the dotted line is the analytical values for the model at $T_{I C}$. The estimated behaviors are $\left\langle E^{2}\right\rangle-\langle E\rangle^{2} / L^{2} \sim L^{0.9}$ and $\left\langle M^{2}\right\rangle-\langle M\rangle^{2} / L^{2} \sim L^{1.8}$ (the error on the exponents is on the last digit given). Where not shown, the errors are included in the symbols; all quantities are dimensionless [10].

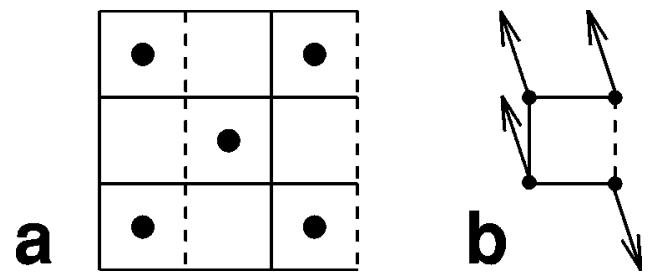

FIG. 4. Square FF lattice: the solid (dashed) lines are ferromagnetic (antiferromagnetic) interactions. (a) Checkerboard partition of a square lattice: the plaquettes with (without) a dot give a pattern. (b) An example of plaquette with three satisfied interactions: In this case we activate the two vertical bonds.

Ising spin-glass (SG) model could allow one to sample very efficiently the equilibrium spin configurations of the system at $T=T_{p}$, where the KF-CK clusters percolate [in a twodimensional (2D) SG $T_{p} \simeq 1.8[4]$ and in a $3 \mathrm{D} \mathrm{SG} T_{p} \simeq 3.95$ [12]] with a dynamics that exhibits self-organized criticality. However since $T_{p}$ is usually much larger than the spin-glass critical temperature $T_{S G}$ (in a 2D SG $T_{S G}=0$ and in a 3D SG $T_{S G} \simeq 1.11$ [13]), any other dynamics does not suffer for slowing down.

\section{IC DYNAMICS FOR THE FF ISING MODEL WITH KBD CLUSTERS}

We ask now how to build up an IC dynamics able to drive a system to the frustrated thermodynamical critical temperature. From the analysis and the argument given for the ferromagnetic case we understand that the cluster definition needs to be modified in such a way that (i) the frustrated system can still be mapped onto the corresponding correlated percolation model and (ii) the clusters percolate at the thermodynamical critical temperature [14]. The first condition is always satisfied by the KF-CK clusters [15], but not the second one. A general procedure to construct such clusters in a systematic way was suggested in Ref. [5]. In particular, for the FF model this procedure leads to the cluster algorithm [6] proposed by Kandel, Ben-Av, and Domany [16]. To define the clusters in KBD dynamics one partitions the square lattice in a checkerboard way and chooses randomly one of the two patterns [Fig. 4(a)]. For each plaquette, if three of four spin pairs are satisfied (a single plaquette in the FF model can have either one or three satisfied spin pairs) one activates bonds between the two spin pairs satisfying the interaction and facing each other [Fig. 4(b)] with a probability $p=1-\exp (-4 / T)$. Numerically it was shown [17] that the clusters obtained with such a procedure percolate at the thermodynamical critical temperature $T_{c}=0$, with critical exponents $\nu_{p}=1$ and $\gamma_{p}=2$ to be compared with the thermodynamical critical exponents $\nu=1$ and $\gamma=3 / 2$.

With this idea in mind we propose the following invaded cluster dynamics. From the checkerboard partition we order randomly all the square plaquettes belonging to the chosen pattern. Then plaquettes are tested in this order to see how many spin pairs are satisfied and in plaquettes with three spin pairs satisfying the interaction we activate bonds between the two spin pairs satisfying the interaction and facing each other. Every time a pair of bonds is activated the cluster structure changes and the occurrence of a spanning cluster is checked. As in the previous cases, when the first cluster per- 


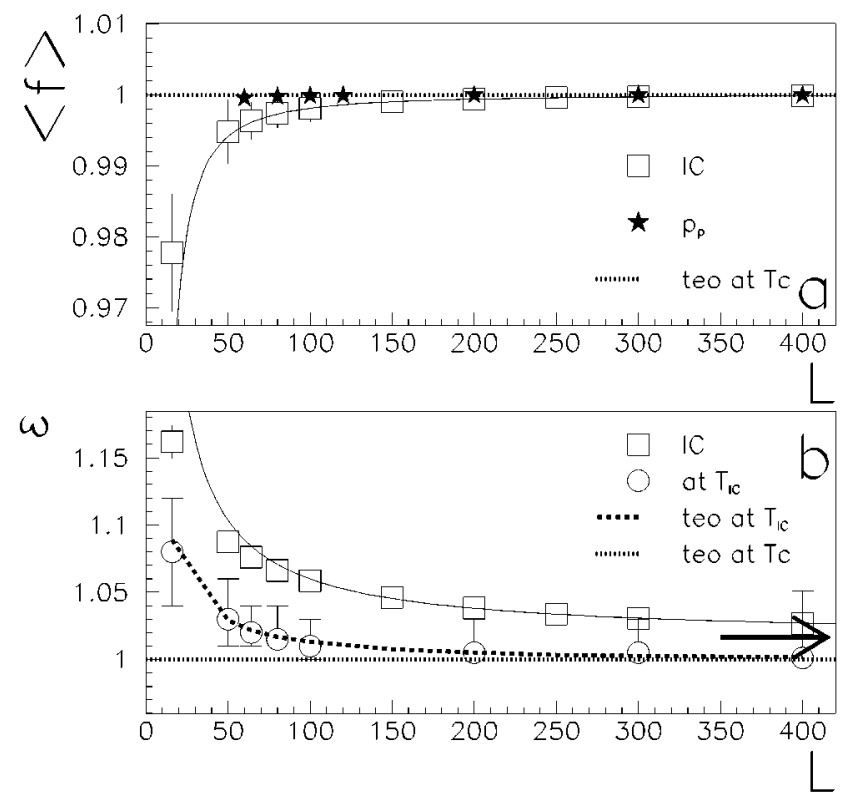

FIG. 5. KBD clusters: (a) ratio of plaquettes with activated bonds to plaquettes with three satisfied interactions $\langle f\rangle$ and (b) energy density $\epsilon$ versus system size $L$ for a square FF system. Squares are the IC dynamics output and the superimposed lines are the plot of the fit functions $\langle f\rangle=\langle f\rangle_{\infty}-A / L-B / L^{2}$ (with $\langle f\rangle_{\infty}=1.000 \pm 0.003, \quad A=0.1 \pm 1.2, \quad$ and $\left.\quad B=9 \pm 100\right) \quad$ and $\epsilon=\epsilon_{\infty}-A / L-B / L^{2}$ (with $\epsilon_{\infty}=1.016 \pm 0.002, A=4.3 \pm 0.5$, and $B=1.0 \pm 0.7)$ for $L \geqslant 100 ;$ stars are the values of $p_{p}=1-\exp \left[-4 / T_{p}(L)\right]$ with $T_{p}(L)$ percolation temperature of KBD clusters in a square FF system of size $L$ (from Ref. [17]); circles are the energy density values of KBD dynamics at the estimated temperature $T_{I C}$ in Table II (the errors are asymmetric because they are derived from the indetermination of $T_{I C}$ ); the dashed line is the analytical energy density for the model at $T_{I C}$; the dotted line is the asymptotic value at $T_{c}=0$; the arrow shows the asymptotic value estimated by the fit. Where not shown, the errors are included in the symbols; all quantities are dimensionless [10].

colates the cluster evolution is stopped and a new spin configuration is obtained by reversing the spin belonging to each cluster altogether with probability $1 / 2$. The dynamics proposed is related to the algorithm introduced by Kandel, BenAv, and Domany exactly as the IC dynamics by Machta et al. is related to the SW algorithm.

TABLE II. KBD clusters: numerical estimates of $T_{I C}(L)$ [10].

\begin{tabular}{ccc}
\hline \hline$L$ & $T_{I C}(L)$ & Error \\
\hline 16 & 1.05 & 0.15 \\
50 & 0.76 & 0.17 \\
64 & 0.71 & 0.12 \\
80 & 0.67 & 0.12 \\
100 & 0.64 & 0.14 \\
150 & 0.58 & 0.16 \\
200 & 0.54 & 0.19 \\
250 & 0.51 & 0.19 \\
300 & 0.49 & 0.25 \\
400 & 0.46 & 0.46 \\
\hline \hline
\end{tabular}

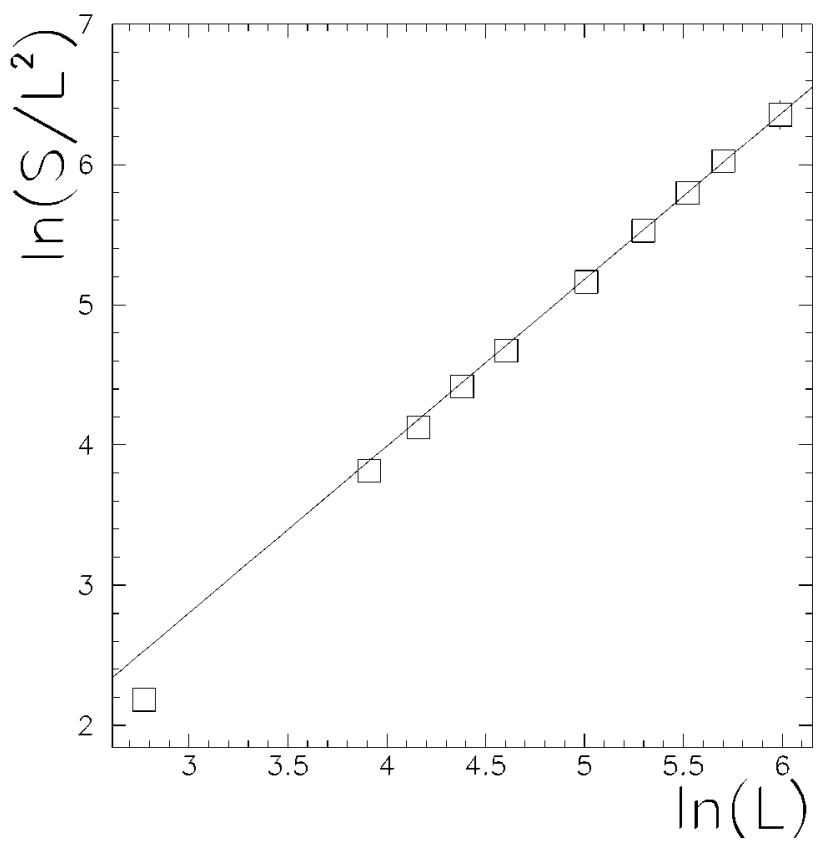

FIG. 6. KBD clusters: mean cluster size per spin $S / L^{2}$ for IC dynamics for a square FF system with $L=16-400$. We show the linear fit $\ln \left(S / L^{2}\right)=A+\gamma_{p} / \nu_{p} \ln (L)$ over data points for $L \geqslant 250$ with $A=-0.8 \pm 1.5$ and $\gamma_{p} / \nu_{p}=1.2 \pm 0.2$. The errors are included in the symbols; all quantities are dimensionless [10].

We have tested the proposed algorithm performing measurements over $10^{4} \mathrm{MC}$ sweeps on square lattices of sizes $L$ ranging from 16 to 200 and over $5 \times 10^{3}$ MC sweeps on $L=250-400$, after discarding the first $10^{3}$ for equilibration. In Fig. 5(a) we show the results of our simulations, which give a situation very similar to that obtained in the ferromagnetic case. In fact, the ratio of plaquettes with activated bonds $N_{b}$ to plaquettes with three satisfied interactions $N_{s}=\left(3 L^{2}-E\right) / 4,\langle f\rangle=N_{b} / N_{s}$, reaches the value $p_{c}=1$ for large system size $L$. At the same time the effective temperature $T_{I C}$ (see Table II), obtained by $\langle f\rangle=1-\exp \left(-4 / T_{I C}\right)$, converges rapidly to the limit value $T_{c}=0$. In Fig. 5(a) we shows also $p_{p}=1-\exp \left[-4 / T_{p}(L)\right]$, where $T_{p}(L)$ is the estimated [17] percolation temperature of KBD clusters in a square FF system of size $L$. The mean energy per spin [Fig. 5(b)] $\epsilon$ tends, within the numerical precision obtained, towards the thermodynamic value at the critical temperature $\epsilon(T=0)=1$ with the expected size dependence $\epsilon-\epsilon_{\infty}$ $=A L^{-1}+B L^{-2}$. The fit of the data gives $\epsilon_{\infty}=1.016 \pm 0.002$. The errors on $\langle f\rangle$ and $\epsilon$ go to zero with increasing $L$. The estimated mean cluster size exponents $\gamma_{p} / \nu_{p}=1.2 \pm 0.2$ (Fig. 6) do not coincide with the expected values $\gamma_{p} / \nu_{p}=2$ [17]. We explain this result with the very slow convergence as a function of $L$ of the percolation quantities obtained with the KBD clusters (see, for example, Fig. 8 of Ref. [17]). Thus we expect to recover the right behavior only for fairly large sizes. We have studied the energy and magnetization fluctuations also in this case (Fig. 7) and found that $\left\langle E^{2}\right\rangle-\langle E\rangle^{2} \sim L^{2.1}$ and $\left\langle M^{2}\right\rangle-\langle M\rangle^{2} \sim L^{3.1}$. These exponents, as in the previous cases, do not agree with those expected for the specific heat and magnetic susceptibility. As in the fer- 

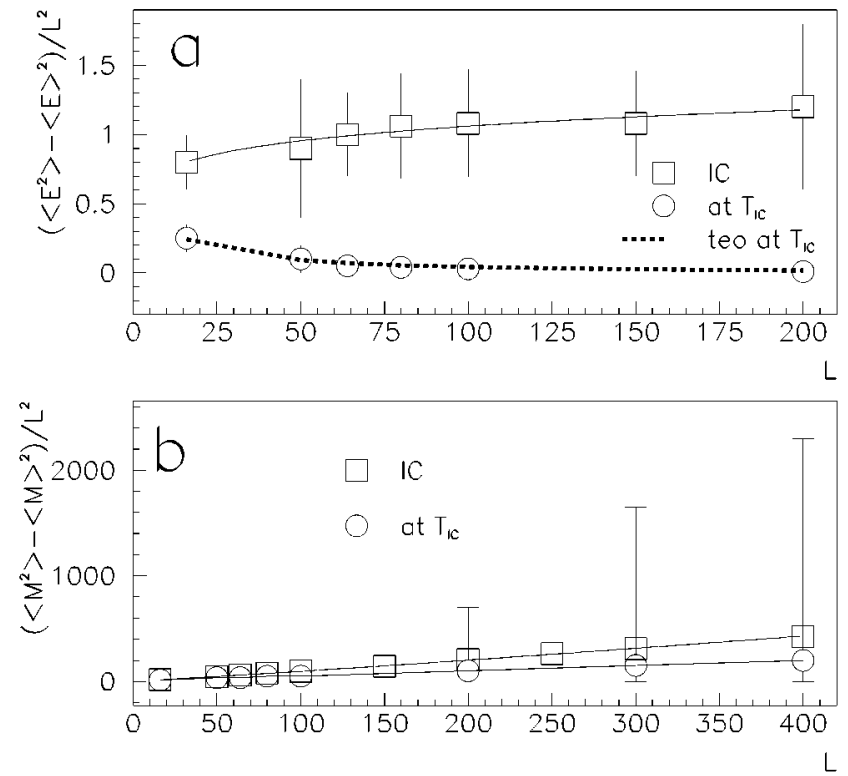

FIG. 7. KBD clusters: the density of (a) the energy fluctuation and (b) the magnetization fluctuation for a square FF system. Squares are the fluctuations for IC dynamics; circles are the fluctuation values of a standard MC dynamics at the estimated temperature $T_{I C}$ in Table II (the errors are asymmetric because they are derived from the indetermination of $T_{I C}$ ); the dashed line is the analytical values for the model at $T_{I C}$. The estimated behaviors are $\left\langle E^{2}\right\rangle-\langle E\rangle^{2} / L^{2} \sim L^{0.1}$ and $\left\langle M^{2}\right\rangle-\langle M\rangle^{2} / L^{2} \sim L^{1.1}$ (the error on the exponents is on the last digit given). Where not shown, the errors are included in the symbols; all quantities are dimensionless [10].

romagnetic case, we obtain that energy and magnetization fluctuations are larger in the IC dynamics than in the canonical ensemble.

We have also studied the equilibrium relaxation of the

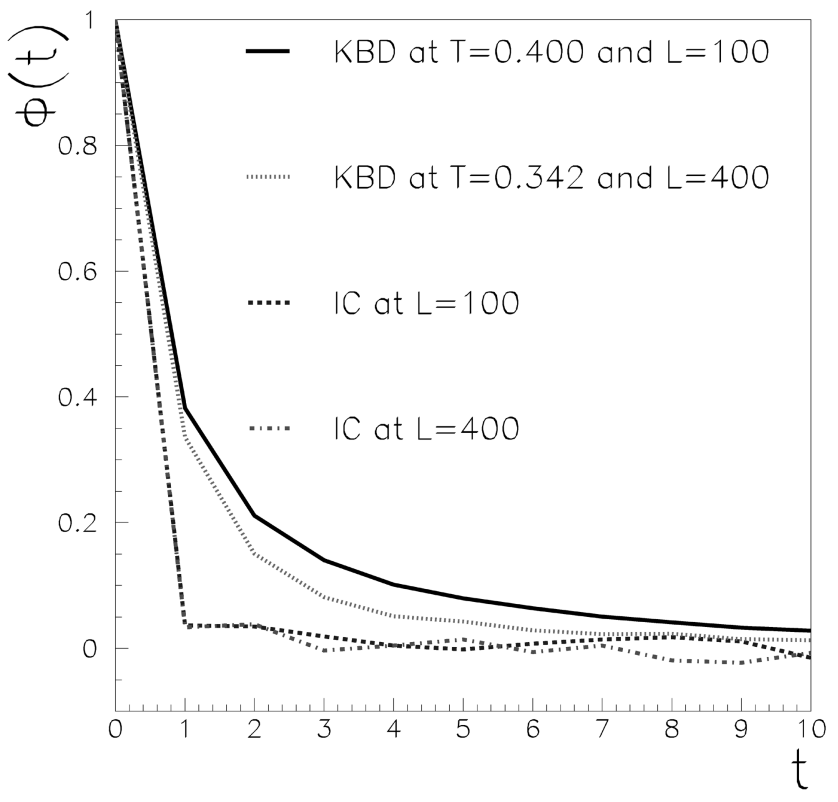

FIG. 8. Magnetization correlation functions in a square FF system for the KBD dynamics [for $L=100$ at $T \simeq T_{p}(L) \simeq 0.45$ and for $L=400$ at $T=T_{p} \simeq 0.342$ [17]] and for IC dynamics with KBD clusters (for $L=100$ and $L=400$ ). Time $t$ is measured in MC steps.
TABLE III. Integrated autocorrelation time $\tau$ for IC dynamics with KBD clusters and $\tau_{K B D}$ for KBD dynamics.

\begin{tabular}{ccc}
\hline \hline$L$ & $\tau$ & $\tau_{K B D}$ \\
\hline 100 & $1.74 \pm 0.02$ & $2.800 \pm 0.008$ \\
200 & $1.73 \pm 0.02$ & $2.48 \pm 0.02$ \\
300 & $1.67 \pm 0.02$ & $2.56 \pm 0.01$ \\
400 & $1.60 \pm 0.02$ & $2.37 \pm 0.03$ \\
\hline \hline
\end{tabular}

magnetization of the proposed IC dynamics

$$
\phi(t)=\frac{\left\langle M\left(t^{\prime}\right)^{2} M\left(t^{\prime}+t\right)^{2}\right\rangle-\left\langle M\left(t^{\prime}\right)^{2}\right\rangle^{2}}{\left\langle M\left(t^{\prime}\right)^{4}\right\rangle-\left\langle M\left(t^{\prime}\right)^{2}\right\rangle^{2}},
$$

where the time $t$ is measured in MC steps. As shown in Fig. $8, \phi(t)$ vanishes in a few MC steps. The integrated autocorrelation time $\tau$, defined as

$$
\tau=\frac{1}{2}+\sum_{t=1}^{t_{m}} \phi(t)
$$

is reported in Table III for different values of $L$. The dependence on $t_{m}$ is extremely weak since $\tau$ reaches a plateau very quickly. The value $\tau \sim 1.6$ obtained is lower than that obtained in the KBD dynamics $\tau_{K B D} \sim 2.4$ and shows a weak tendency to decrease with increasing $L$.

\section{CONCLUSIONS}

We have shown how the IC dynamics introduced by Machta et al. for the ferromagnetic Ising model can be extended to the FF Ising model on a square lattice. The straightforward extension with KF-CK clusters shows that the IC dynamics leads to a self-organized critical percolating state at the percolation temperature $T_{p}$ and can be used to produce equilibrium spin configurations at a temperature different from the thermodynamical critical temperature, actually at the percolation temperature $T_{p}$. The dynamics is characterized by intrinsic diverging fluctuations and the fluctuation-dissipation theorem is no longer valid. The extension with KBD clusters, whose percolation point coincides in the large- $L$ limit with the critical point of the FF system, has properties very similar to those obtained in the ferromagnetic model: It drives the system to the critical region without previous knowledge of the critical temperature and gives a reasonably good estimation of the average energy at the critical point. The estimated integrated autocorrelation time is smaller than that obtained in the KBD dynamics. We have also stressed that the extension is possible since there exists a percolation model onto which the FF square Ising model can be exactly mapped. The extension to other frustrated systems, such as spin glasses, in principle, can be done using the systematic procedure suggested in Ref. [5]. The computation was done on a DEC station 3000/500 with an Alpha processor. 
[1] J. Machta, Y. S. Choi, A. Lucke, and T. Schweizer, Phys. Rev. Lett. 75, 2792 (1995).

[2] C. M. Fortuin and P. K. Kasteleyn, Physica (Amsterdam) 57, 536 (1972); A. Coniglio and W. Klein, J. Phys. A 15, 1873 (1980).

[3] R. H. Swendsen and J. S. Wang, Phys. Rev. Lett. 58, 86 (1987).

[4] V. Cataudella, Physica A 183, 249 (1992).

[5] V. Cataudella, G. Franzese, M. Nicodemi, A. Scala, and A. Coniglio, Phys. Rev. Lett. 72, 1541 (1994); Phys. Rev. E 54, 175 (1996).

[6] D. Kandel, R. Ben-Av, and E. Domany, Phys. Rev. Lett. 65, 941 (1990); D. Kandel and E. Domany, Phys. Rev. B 43, 8539 (1991).

[7] J. Machta, Y. S. Choi, A. Lucke, T. Schweizer, and L. M. Chayes, Phys. Rev. E 54, 1332 (1996).

[8] T. B. Liverpool and S. C. Glotzer, Phys. Rev. E 53, R4255 (1996).

[9] G. Johnson, H. Gould, J. Machta, and L. K. Chayes, cond-mat/9704163.

[10] Henceforth we choose $k_{B}=J=1$ such that the temperature $T=k_{B} T / J$ and energy $E=E / J$ are dimensionless. Furthermore, we choose unitary lattice space $a$ and unitary Bohr magneton $\mu_{B}$ such that the linear lattice size $L=L / a$ and the magnetization $M=M / \mu_{B}$ are also dimensionless. In this way all quantities considered are dimensionless unless specified otherwise.

[11] D. Stauffer and A. Aharony, Introduction to Percolation
Theory, 2nd ed. (Taylor \& Francis, London, 1992).

[12] L. de Arcangelis, A. Coniglio, and F. Peruggi, Europhys. Lett. 14, 515 (1991).

[13] N. Kawashima and A. P. Young, Phys. Rev. B 53, R484 (1996).

[14] The two conditions (i) and (ii) have been discussed in detail in Ref. [5]. In general, these two conditions are not sufficient to ensure that percolation clusters represent spin-correlated regions, i.e., to ensure that the set of percolation critical exponents coincides with the set of thermodynamical critical exponents ( $\nu_{p}=\nu, \gamma_{p}=\gamma$, and so on). However, in many cases it has been shown [5] that these two conditions allow a dramatic decrease of the critical slowing down.

[15] A. Coniglio, F. di Liberto, G. Monroy, and F. Peruggi, Phys. Rev. B 44, 12605 (1991).

[16] In Ref. [5] a FF model (called the asymmetric FF model) has also been studied numerically where the antiferromagnetic interaction strength is $X$ times the ferromagnetic interaction strength, with $0 \leqslant X<1$, showing that it is always possible to find a cluster definition (different from the KF-CK and KBD cluster definitions) such that $T_{p}=T_{c}, \nu_{p}=\nu, \gamma_{p}=\gamma$, and so on, within the numerical precision. Let us note that in this paper we are considering the symmetric FF model $(X=1)$ where only $T_{p}=T_{c}$ and $\nu_{p}=\nu$ still hold within the numerical precision (see the text).

[17] G. Franzese, J. Phys. A 29, 7367 (1996). 